\title{
Ruralité en péril : redéfinir les consensus et argumentaires en faveur de l'occupation dynamique des territoires fragiles
}

\author{
Bernard Vachon 1 \\ Université du Québec à Montréal
}

Malgré que le déclin économique et démographique semble avoir atteint un stade irréversible dans plusieurs communautés rurales des régions éloignées et intermédiaires, la mise en place des conditions nécessaires à une relance durable du développement de ces régions demeure un objectif de société réaliste. Des politiques volontaristes énergiques mieux adaptées et mieux ciblées, confortées par une solidarité territoriale entre régions riches et régions pauvres, pourraient briser la fatalité du déclin en territoires fragiles. Pour y arriver, un vrai débat doit s'ouvrir sur la place des régions périphériques et intermédiaires dans le développement de la société québécoise, sur la nature et l'ampleur des investissements structurants à réaliser, sur les coûts d'une occupation dynamique des régions en difficulté et sur l'effort susceptible d'être consenti par les régions centrales.

Comme préalable, les consensus et argumentaires qui ont prévalu jusqu'à présent en matière de développement régional et de développement rural feront l'objet d'une entière révision à la lumière d'une mise à jour de l'état des lieux.

\section{Une gouvernance territoriale qui consolide la tendance lourde de la concentration}

On a beaucoup parlé des régions et de la ruralité au cours des dix-huit derniers mois au Québec. En décembre 2001, le gouvernement du Québec adoptait en grande pompe une Politique nationale de la ruralité et moins d'un an plus tard il organisait le Rendez-vous national des régions. Le ciel est-il maintenant plus serein pour les régions non centrales maillées d'un réseau de petites villes et de villages? C'est à regret qu'il faut convenir que non, car au-delà des beaux discours et des enga- gements la main sur le coeur en faveur des régions, très peu fut offert et très peu sera fait. La Politique de la ruralité est une déclaration d'attachement à l'égard des communautés rurales et de leur différence, accompagnée d'un modeste budget de 90 millions \$ pour la réalisation de projets locaux pour les 1000 municipalités rurales au cours des cinq prochaines années; faites le calcul. On a dit de cette politique que c'était un premier pas non négligeable. Peut-être, mais à quand les pas majeurs? Sans arrimage à une vigoureuse politique de développement régional, la politique de la ruralité aura peu d'effet à moyen et long termes.

\section{Sans arrimage à une vigoureuse politique de développement régional, la politique de la ruralité aura peu d'effet à moyen et long termes.}

L'appel au Rendez-vous national des régions avait laissé croire un temps que les assises d'une véritable politique de développement régional seraient peut-être édifiées. Point n'en fut! Des bricoles furent accordées, des décisions furent reportées, des comités furent créés. On se permit même un nouveau recul sur la décentralisation: par ici la «décentralisation à la carte»!

$\mathrm{Du}$ point de vue gouvernemental, le succès de ce Rendez-vous est dans l'apaisement des régions. La paix est à nouveau rétablie sans qu'il en ait coûté trop cher et surtout sans que des transferts de budgets des régions centrales vers les régions éloignées n'aient été nécessaires. L'observateur constate l'habilité du gouvernement à obtenir cet apaisement avec des parades et bien peu d'investissements consentis; constate aussi la reddition des troupes régionales sans forte résistance en échange 
de bien maigres gains de négociations. Le mot d'ordre est de régler les tensions en régions excentrées sans qu'il n'en coûte trop cher et sans contrarier les grands centres.

Du point de vue gouvernemental, le succès de ce Rendez-vous est dans l'apaisement des régions. La paix est à nouveau rétablie sans qu'il en ait coûté trop cher et surtout sans que des transferts de budgets des régions centrales vers les régions éloignées n'aient été nécessaires.

Dans sa réforme municipale (2001), la ministre Louise Harel a été principalement motivée par la consolidation des 31 agglomérations du réseau urbain du Québec, incluant les trois zones métropolitaines de Montréal, Québec et Hull. Son credo, partagé largement par le gouvernement, était que le développement du Québec passe d'abord et avant tout par des villes fortes, rejoignant en cela la thèse économique de la concentration spatiale du capital et des populations ayant pour corollaire le dépérissement progressif et inévitable des régions non centrales. Le résultat a été, en définitive, une politique de la ville. On peut imaginer que cette réforme aurait pu être un merveilleux instrument au service d'une Politique territoriale nationale. Négligés par les grands desseins de l'État, les territoires ruraux qui composent l'arrièrepays de l'armature urbaine se sentent de plus en plus excédentaires, résiduels, révolus et indésirables dans un contexte où l'essor de la société accorde bien peu de place à leur contribution. Leur destin s'inscrit dans un mouvement où extinction et disparition sont, à terme, dans l'ordre des choses.

Négligés par les grands desseins de I'État, les territoires ruraux qui composent l'arrière-pays de l'armature urbaine se sentent de plus en plus excédentaires, résiduels, révolus et indésirables dans un contexte où l'essor de la société accorde bien peu de place à leur contribution. Leur destin s'inscrit dans un mouvement où extinction et disparition sont, à terme, dans l'ordre des choses.
Les villes de Montréal et de Québec continuent d'être les lieux privilégiés d'investissement en termes d'infrastructures: prolongement de l'autoroute 30 au sud de Montréal (730 M\$), revitalisation des quartiers à Québec et à Montréal (85,7 $\mathrm{M} \$)$, implantation d'un réseau de tramway à Québec $(650 \mathrm{M} \$)$, prolongement du Métro à ville de Laval, etc.

Entre-temps, le service de transport aérien régional continue à se dégrader; le projet de prolongement de l'autoroute 20 entre Rivière-du-Loup et Mont-Joli est dans l'attente de son parachèvement depuis près de vingt-cinq ans; la voirie tertiaire de très nombreuses municipalités rurales est dans un état lamentable depuis le retrait du gouvernement provincial de ce champ de responsabilité (réforme Ryan); le service de traversier entre Trois-Pistoles et Les Escoumins est suspendu faute d'entretien du quai des Escoumins par le gouvernement fédéral, un lien inter-rives pourtant stratégique pour l'activité économique de deux régions du Québec maritime, etc.

\section{Le toujours lancinant dépérissement rural}

Il y a près de 1000 municipalités rurales au Québec, dont 602 de 800 habitants et moins et 224 de 300 habitants et moins (recensement de 2001). Des centaines d'entres elles voient fondre et vieillir leurs populations depuis les années 1960 et atteindre des seuils qui menacent désormais leur survie. Leurs réserves de vitalité économique, démographique et sociale en expansion jusqu'au début des années 1960, se sont épuisées depuis. Les jeunes partent, les populations restantes vieillissent à un rythme accéléré, les champs désertés sont reboisés, les commerces ferment, l'environnement bâti se dégrade, la diversification économique salvatrice n'est pas au rendez-vous. On entretient l'espoir, mais celui-ci se languit dans une trop longue attente. Le temps est peut-être venu de reconnaître que malgré la pertinence intrinsèque du discours ruraliste, l'aberration de la désertion des régions rurales et le caractère louable des quelques efforts consentis dans le cadre de politiques de l'État, et par des partenaires territoriaux, toutes les communautés rurales ne pourront prendre le train de la «modernité rurale». Et il est à prévoir que pour plusieurs d'entre elles, le processus de dévitalisation, désormais irréversible, va se poursuivre jusqu'à leur extinction. 


\section{Le temps est peut-être venu de reconnaître que malgré la pertinence intrinsèque du discours ruraliste, l'aberration de la désertion des régions rurales et le caractère louable des quelques efforts consentis dans le cadre de politiques de l'État, et par des partenaires territoriaux, toutes les communautés rurales ne pourront prendre le train de la umodernité rurale ". Et il est à prévoir que pour plusieurs d'entre elles, le processus de dévitalisation, désormais irréversible, va se poursuivre jusqu'à leur extinction.}

Récemment, Murdochville a été un témoin hautement médiatisé de cette réalité des petites villes et villages en sursis que l'on constate mais que l'on refuse d'admettre. La perte de sa vocation minière d'origine l'a précipitée au bord de l'abîme. L'enjeu de sa survie est la reconversion de son économie vers des créneaux gagnants. Mais lesquels? Des dizaines de municipalités rurales, après le déclin progressif de leur économie agro-forestière ou minière ou de pêches, sont aujourd'hui dans la même situation à travers le Québec des régions périphériques et intermédiaires (le cas d'Asbestos est actuellement sur la sellette avec la fermeture annoncée de l'usine de transformation du magnésium, Magnolia, projet porteur de grands promesses lors de sa réalisation il y a moins de trois ans). Dans un tel contexte, Murdochville et Asbestos deviennent des laboratoires expérimentaux pour la prise de décisions politiques à l'égard des villages et des petites villes qui se meurent et pour la mise au point de stratégies «adaptées » pour des régions victimes de marginalisation, voire d'exclusion, suite aux bouleversements qui traversent l'économie nationale et mondiale. Les résultats peu probants issus jusqu'à présent de ces «laboratoires », comme de celui de la Gaspésia et de tant d'autres, confirment une fois de plus la fragilité économique et démographique des régions non centrales et la difficulté multivariée de gérer cette réalité, tant pour contrer sa marche que pour encadrer ses conséquences dramatiques au sein des populations locales.

Des territoires qui meurent, c'est d'abord le résultat de changements importants et durables dans les rapports de l'activité économique avec l'espace. Et ces changements, qualifiés de structurels, qui se manifestent par l'évolution de la logique de localisation des investissements de production vers un modèle de plus en plus spatialement concentré, se trouvent conjugués à des tendances sociales lourdes qui empruntent les mêmes voies. Ainsi, la concentration des moyens de production et de la population sur quelques lieux ou régions centrales, s'accompagne de la contraction de l'espace habité. Vis-àvis de ces forces extérieures aux territoires, les compétences et aptitudes collectives locales et régionales ont un impact bien limité (les ressources naturelles n'échappent pas à cette règle, les déboires récents dans les domaines des pêches, du bois d'œuvre et des mines en témoignent éloquemment). Les profonds chambardements qui découlent des mutations structurelles se traduisent dans l'organisation du territoire par la double réalité des «régions qui gagnent et des régions qui perdent ${ }^{2}$. Voilà le schéma général.

\section{Ainsi, la concentration des moyens de production et de la population sur quelques lieux ou régions centrales, s'accompagne de la contraction de l'espace habité.}

Face à cette réalité que peu d'observateurs contestent, plusieurs questions peuvent être soulevées, notamment des questions relatives aux consensus et argumentaires en faveur de la relance des régions excentrées et des territoires ruraux fragiles qui sont à la source de beaucoup de prises de positions et d'interventions à l'égard de ces milieux.

\section{Consensus et argumentaire en faveur du renouveau rural}

Depuis le milieu des années 1960, suite notamment aux fermetures décrétées de villages en Gaspésie et aux mouvements de résistance baptisés «Dignité I » et «Dignité II» qu'elles suscitèrent au sein des populations locales, des consensus favorables à l'édification d'une ruralité nouvelle se sont progressivement construits et solidement implantés. Syndicats d'agriculteurs et de forestiers, chercheurs universitaires, groupes écologistes, chambres de commerce, élus locaux et associations diverses se sont portés à la défense de la ruralité, prônant les valeurs et les mérites d'une occupation dynamique du territoire rural, non seulement pour les populations qui y vivent mais pour l'essor de la société québécoise toute entière. Souvent émotifs à l'origine, les consensus ont évolué vers une réflexion globale et une prise en compte d'une société en pleine mutation (les États généraux du 
monde rural tenus en 1991 ont constitué une étape très importante à cet égard). Il en est résulté un argumentaire aux multiples volets prônant la diversification économique, un mode d'emploi élargi des territoires ruraux et un nouveau rapport ville-campagne, ouvrant des perspectives stimulantes pour l'avenir du monde rural. Les scénarios les plus progressistes ont été élaborés et proposés pour assurer une «occupation dynamique du territoire rural».

L'argumentaire en faveur de la «renaissance rurale» repose aussi sur la capacité des communautés rurales à saisir les opportunités de développement procurées par des changements survenus plus récemment dans la logique de localisation de plusieurs secteurs d'activité et de travailleurs: il s'agit d'un mouvement centrifuge d'activités économiques et de personnes, parallèlement au mouvement de concentration général qui se poursuit. Ce phénomène se décline en fonction des réalités nouvelles suivantes: 1) le potentiel de desserrement, voire de déconcentration, d'une part non négligeable de la trame économique, du fait de la dématérialisation d'un nombre croissant de ses activités et systèmes de production; 2) l'adhésion à des mesures de diversification des économies locales et régionales ouvrant la porte aux entreprises de deuxième et de troisième transformation à plus forte valeur ajoutée; 3) le développement et la généralisation des nouvelles technologies d'information et de communication comme facteur d'éclatement des lieux de production et de travail; 4) l'intérêt pour une meilleure qualité de vie chez une partie croissante de la population et 5) les revendications territoriales pour une décentralisation accrue des pouvoirs et des leviers de développement.

\section{Une renaissance rurale qui tarde à se manifester}

\section{Des seuils de population qui rendent difficile le maintien des commerces et des services de base, ce qui mine la capacité de rétention et d'attraction de ces communautés.}

Malgré certains progrès enregistrés, six ensembles de circonstances font obstacle à l'avènement attendu de la «renaissance rurale», particulièrement dans les régions périphériques et intermédiaires compromettant l'avenir de nombreuses municipalités rurales : 1) le contexte nouveau générateur d'opportunités de développement pour le monde rural (desserrement économique et démographique) se met en place à un rythme nettement plus lent que le caractère d'urgence, qui prévaut dans un grand nombre de communautés rurales, le requiert; 2) la faible natalité combinée à l'exode des jeunes, au vieillissement accéléré des populations restantes et à l'apport négligeable de nouveaux résidents (mouvements interrégionaux et immigration), conduisent de nombreuses communautés à des seuils de population qui rendent difficile le maintien des commerces et des services de base, ce qui mine le capacité de rétention et d'attraction de ces communautés; 3 ) l'effritement de la volonté et de la capacité collective à lutter contre le déclin économique et social nuit à la prise en charge locale et empêche l'émergence d'idées innovantes indispensable au renouveau rural; 4) les grandes villes du Québec n'ont pas ce haut niveau de dysfonctionnement (pollution, insécurité, congestion de la circulation...) qui les rendrait invivables; au contraire, elles sont parmi les villes d'Amérique du nord offrant la plus haute qualité de vie, ce qui met en échec l'argument «qualité de vie» mis de l'avant par certaines régions éloignées et intermédiaires pour attirer de nouvelles familles et entreprises; 5) l'habilité et l'efficacité des stratégies des régions centrales de Montréal et de Québec à convaincre les gouvernements fédéral et provincial d'investir massivement et en priorité sur leur territoire et enfin, 6) les politiques nationales d'investissements structurants hors des régions centrales ne sont pas à la hauteur des défis que pose le développement des régions rurales périphériques et intermédiaires.

\section{Les politiques nationales d'investissements structurants hors des régions centrales ne sont pas à la hauteur des défis que pose le développement des régions rurales périphériques et intermédiaires.}

Si bien que l'écart s'accentue entre les territoires ruraux sous l'influence des grands centres (Montérégie, Lanaudière, Laurentides et Capitale nationale principalement) et ceux des régions périphériques et intermédiaires. Une situation qui questionne et qui réclame de nouveaux consensus. Y a-t-il danger que le degré de dévitalisation qui affecte nombre de communautés rurales en régions éloignées soit désormais trop avancé, les populations trop affaiblies, la cohésion sociale trop déstructurée et la conjoncture du développement 
territorial encore trop «métropolitaine» pour que les situations qui ne cessent de se détériorer soient renversées? Y a-t-il risque que les discours et les interventions pour «maintenir l'espoir» dans les communautés en difficulté prennent dorénavant l'allure de pures illusions pour plusieurs d'entre elles? Selon quels critères peut-on conclure à l'irréversibilité du déclin d'une localité? Face à la menace d'extinction, jusqu'où l'assistance de l'État doit-elle être assurée avant l'issue fatale et quelle forme doit-elle prendre? Peut-on, comme société, envisager et gérer rationnellement le processus de contraction de l'espace occupé? Si oui, comment?

\section{Y a-t-il risque que les discours et les inter- ventions pour " maintenir l'espoir » dans les communautés en difficulté prennent dorénavant l'allure de pures illusions pour plusieurs d'entre elles?}

\section{Revoir la rhétorique et les consensus relatifs à l'avenir des territoires ruraux}

Depuis le milieu des années 1980, un formidable mouvement «ruraliste» s'est développé au Québec animé par une forte volonté de sauver les campagnes. Aux constats alarmants d'organismes publics, aux manifestations populaires, aux résultats de la recherche universitaire, aux revendications territoriales, aux «scénarios gagnants», au modèle des «villages prospères », les gouvernements ont répondu par la mise en place de structures officielles d'intervention locale. $\mathrm{Ne}$ citons ici que les SADC ${ }^{3}$, les CLD ${ }^{4}$, le mouvement Solidarité Rurale 5 et la Politique nationale de la ruralité. Malgré le bien-fondé de ces structures et les services rendus aux populations des territoires concernés, il faut reconnaître aujourd'hui que dans plusieurs milieux, les résultats sont minces en regard des attentes et de l'ampleur des défis à relever: les gains obtenus, au prix de maints efforts, ne parviennent pas à juguler les forces destructrices qui se poursuivent et qui s'annoncent comme irréversibles dans bien des cas. Une large part du travail de réflexion, d'intervention et d'accompagnement poursuivi au sein de ces structures vise à «entretenir l'espoir », ce qui signifie pour nombre de communautés rurales, retarder l'échéance de l'extinction. Bien sûr, de belles réalisations et des «success stories» sont à porter au crédit de ces organismes et des initiatives du milieu, mais ce n'est pas suffisant pour renverser la situation. Les tendances lourdes qui se poursuivent érodent inlassablement le cadre général de développement et affaiblissent la volonté et la capacité endogènes des communautés locales à participer activement au processus de leur développement.

Il faut reconnaître aujourd'hui que dans plusieurs milieux, les résultats sont minces en regard des attentes et de l'ampleur des défis à relever: les gains obtenus, au prix de maints efforts, ne parviennent pas à juguler les forces destructrices qui se poursuivent et qui s'annoncent comme irréversibles dans bien des cas.

Le temps est venu de revoir certains consensus et de "revisiter " l'argumentaire général du renouveau rural. Un regard neuf doit être posé sur "l'état actuel des lieux " et les perspectives inquiétantes qui s'annoncent pour plusieurs communautés rurales.

Le temps est venu de revoir certains consensus et de «revisiter» l'argumentaire général du renouveau rural. Un regard neuf doit être posé sur «l'état actuel des lieux» et les perspectives inquiétantes qui s'annoncent pour plusieurs communautés rurales. Dans un avenir prévisible, les régions centrales vont-elles continuer à retenir leurs populations et leurs activités économiques et conserveront-elles leur puissant pouvoir d'attraction au détriment des régions éloignées et intermédiaires? Est-il réaliste, en 2003, de penser sauver tous les villages du Québec alors que le dépérissement s'accentue d'une part et que la concentration du capital de production et de la population se poursuit d'autre part? En régions périphériques et intermédiaires, le facteur démographique (accroissement naturel et bilan migratoire interrégional) va-t-il continuer à affaiblir les populations locales, tarissant les dernières réserves de vitalité de plusieurs communautés? Les espaces régionaux vont-ils se restructurer autour des villes-centres et d'axes ruraux privilégiés à vocations spécifiques (agricole, récréotouristique, mixte...), abandonnant l'arrière-pays à la forêt et à la friche? L'avenir des régions rurales peut-il dépendre d'un mode d'emploi bio et écolo de leurs 
ressources? Le déclin rural est-il, en partie, le résultat de la faillite de l'imagination des politiques gouvernementales et des initiatives conservatrices du milieu?

Est-il réaliste, en 2003, de penser sauver tous les villages du Québec alors que le dépérissement s'accentue d'une part et que la concentration du capital de production et de la population se poursuit d'autre part? En régions périphériques et intermédiaires, le facteur démographique (accroissement naturel et bilan migratoire interrégional) va-t-il continuer à affaiblir les populations locales, tarissant les dernières réserves de vitalité de plusieurs communautés?

Comme le sort des régions rurales éloignées et intermédiaires dépend largement des tendances lourdes aux niveaux macro-économique et macro-social, les analyses, les politiques et les interventions ne peuvent se limiter au contexte des échelons local et régional. D'autres interrogations se posent: les règles du marchés doivent-elles seules voir à l'organisation du territoire et à la distribution de la population? Jusqu'où la mission sociale de l'État peut-elle être invoquée pour justifier un interventionnisme en faveur des régions en difficulté? Une politique volontariste d'investissements structurants peut-elle infléchir de façon significative la répartition géographique des capitaux de production et des populations et, conséquemment, redessiner la carte de l'occupation du territoire? Quel niveau d'investissements structurants le développement régional requiert-il? Sommes-nous prêts, comme société, à consentir les efforts en faveur d'un meilleur équilibre spatial de l'occupation du territoire? L'appétit insatiable des grands centres urbains en regard des aides de l'état pour favoriser leur croissance conduit-il à négliger non seulement l'essor des régions périphériques et intermédiaires mais également leur rôle dans le développement de la société globale? À défaut de politiques nationales d'envergure en faveur des régions en difficulté, quelles formes privilégiées devrait prendre l'action locale et régionale et quels résultats peut-on espérer de ces interventions?

Les réponses à toutes ces questions vont nécessiter une mise à niveau de la compréhension de la ruralité contemporaine et de ses rapports avec le monde urbain. Elles nécessiteront également de l'imagination créative, du courage politique et une solidarité territoriale. La formulation des nouveaux consensus et des choix appropriés qui en découleront en dépendent. À défaut de quoi, le statu quo irresponsable et les mesures ponctuelles vont faire œuvre de projet. Ce qui peut aussi être un choix !

\section{Une politique volontariste d'investissements structurants peut-elle infléchir de façon significative la répartition géographique des capitaux de production et des populations et, conséquemment, redessiner la carte de l'occupation du territoire? Quel niveau d'investissements structurants le dévelop- pement régional requiert-il? Sommes-nous prêts, comme société, à consentir les efforts en faveur d'un meilleur équilibre spatial de l'occupation du territoire?}

\section{Sans politique vigoureuse de développement régional le développement local est un leurre}

Clermont Dugas, Marc-Urbain Proulx, Bruno Jean, moimême et quelques collègues dans la cinquantaine ou la jeune soixantaine, faisons partie d'une génération de chercheurs ruralistes qui se sont investis sur plus de 20 ou 25 ans dans la compréhension, la défense, la redéfinition et la promotion de la ruralité au Québec (des professionnels du développement territorial et des fonctionnaires de l'État ont aussi contribué à ce mouvement). Passionnés par ce champ d'investigation, nous n'avons négligé aucune forme d'intervention dans notre action: enseignement académique, formation de terrain, rédaction d'ouvrages et d'articles, recherche fondamentale et appliquée, consultation auprès des gouvernements centraux et des instances régionales et locales, appui aux agents de développement, participation à des organismes régionaux, nationaux et internationaux, conférences, séminaires, colloques, université rurale, etc. Une démarche que nous avons privilégiée a été celle du développement local, fondée sur la volonté et la capacité des communautés locales de s'approprier le processus de développement et de générer de l'intérieur leur développement. Notre travail sur ce plan, j'ose le dire, a été exemplaire et il n'a pas été sans influencer l'adoption de la Politique de soutien au développement local et régional, la mise en place des 
réseaux des Sociétés d'aide au développement des collectivités (CADC/SADC) et des Comités locaux de développement (CLD), la pratique de terrain des agents de développement, la mobilisation des populations locales, la mission du mouvement Solidarité rurale et les orientations de la Politique nationale de la ruralité.

Le recours au développement local demeure aujourd'hui toujours pertinent. Il est toutefois plus nécessaire que jamais de rappeler que cette démarche a des limites et que celles-ci sont d'autant plus évidentes et contraignantes dans le contexte actuel que d'une part l'exode et le vieillissement des populations au cours des dernières décennies ont sérieusement appauvri le capital social des zones rurales fragiles et que d'autre part, la poursuite du mouvement de concentration et les timides politiques nationales de développement à visée régionale privent cruellement les régions des infrastructures modernes et suffisantes de support au développement.

\section{Le recours au développement local demeure aujourd'hui toujours pertinent. Il est toutefois plus nécessaire que jamais de rappeler que cette démarche a des limites et que celles-ci sont d'autant plus évidentes et contraignantes dans le contexte actuel que d'une part l'exode et le vieillissement des populations au cours des dernières décennies ont sérieusement appauvri le capital social des zones rurales fragiles et \\ que d'autre part, la poursuite du mouvement de concentration et les timides politiques nationales de développement à visée régionale privent cruellement les régions des infrastructures modernes et suffisantes de support au développement.}

Ces handicaps sévères de l'état des lieux rendent particulièrement difficile la réunion des conditions favorisant les initiatives de développement et la capacité attractive des milieux excentrés à l'égard des capitaux de production et des travailleurs qualifiés, comparés aux régions centrales, pour un développement moderne et durable. Sans une réelle et vigoureuse volonté politique d'assurer l'occupation dynamique du territoire, concrétisée par des programmes majeurs d'investissements en infrastructures, équipements et services appropriés à des niveaux nécessitant des ajustements à la baisse des budgets alloués aux régions centrales, le développement local est condamné à «entretenir l'espoir » et à se cantonner dans des «success stories» ponctuelles et souvent éphémères.

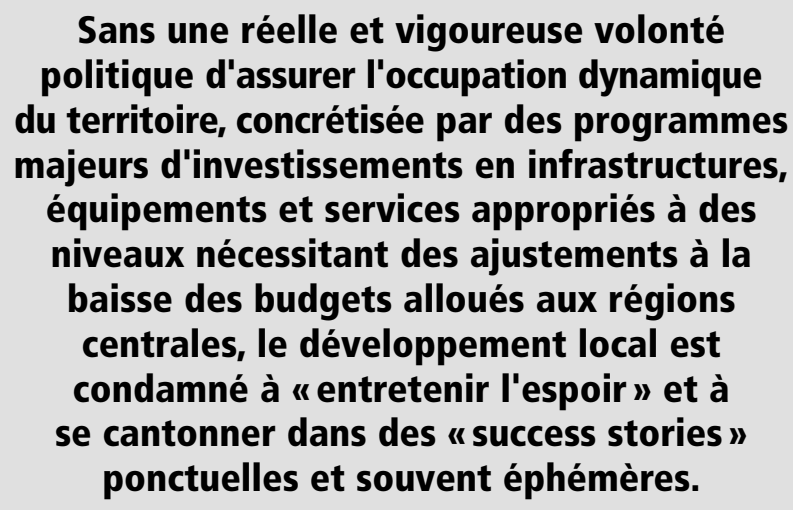

Sans une réelle et vigoureuse volonté politique d'assurer l'occupation dynamique du territoire, concrétisée par des programmes majeurs d'investissements en infrastructures, équipements et services appropriés à des niveaux nécessitant des ajustements à la baisse des budgets alloués aux régions centrales, le développement local est condamné à " entretenir l'espoir " et à se cantonner dans des " success stories » ponctuelles et souvent éphémères.

Ces programmes majeurs d'investissements porteraient sur l'amélioration des réseaux de transport terrestre, maritime et aérien, la formation de la main-d'œuvre dans des secteurs en demande et en émergence, les aides financières et techniques à l'installation de nouvelles entreprises, l'appui au développement de filières de productions privilégiées, l'essor des entreprises de deuxième et de troisième transformation, l'environnement propice à l'implantation des activités de la nouvelle économie, la diversification en agriculture, en foresterie et dans les pêches et la promotion de façons de faire écologiques dans ces domaines, le développement de nouveaux produits touristiques, la disponibilité en logements pour les nouvelles familles ce qui impliquerait plus de souplesse dans l'application du zonage agricole, la rénovation des petites villes et des villages, le maintien des services de proximité incluant les services médicaux de première ligne et la dernière école de village, le service Internet à haute vitesse accessible, selon diverses technologies, sur l'ensemble du territoire habité comme facteur moderne de localisation d'entreprises et de soutien aux travailleurs autonomes (télé-travailleurs), la consolidation des formations de niveaux cégep et universitaire en région, incluant des partenariats avec les milieux économiques et sociaux, les équipements et services récréatifs et culturels diversifiés et faciles d'accès, la protection et la mise en valeur du patrimoine naturel et culturel, etc.

Il faut reconnaître qu'au-delà des beaux discours, aucune des politiques nationales récentes n'a porté ces ambitions aux niveaux nécessaires pour enclencher le développement attendu. Bien que rien ne laisse présager un 
changement significatif à ce chapitre dans un proche avenir, quel que soit le parti politique au pouvoir, il est à souhaiter que la lassitude et le sentiment d'abandon ne conduiront pas à la résignation et à l'abdication. L'avenir des régions en dépend.

\section{Ne pas se méprendre: éteindre les feux de la foudre ne dissipe pas l'orage}

Périodiquement, des situations de crise éclatent en région: fermeture d'usines, suppression de services, pénurie de médecins, épuisement des ressources, déficience des infrastructures de transport, perte massive d'emplois, démantèlement et transfert d'usines de transformation, déficit des équipements culturels, exode des jeunes, etc. Les populations inquiètes maugréent, s'agitent, descendent dans la rue, érigent des barrages, «montent à Québec», se font voir et entendre. Le gouvernement sort alors son «prêt-à-porter anti-agitation » : à grand renfort de caméras, une délégation de ministres se déplace sur le terrain, des discours émotifs sont prononcés, l'avenir du Québec est lié à la résolution de la crise en cours, des engagements qui feront manchettes dans tous les quotidiens sont signés et quelques millions de dollars sortent des chapeaux magiques des ministres. Puis, chacun rentre chez soi jusqu'à la prochaine crise. Au mieux, un certain suivi sera fait du projet ponctuel, qui fait office de solution, et des sommes investies.

Le Rendez-vous des régions de novembre dernier (organisé par le Ministère des Régions) a évité une nouvelle fois le débat de fond sur le sens et le coût d'une occupation dynamique du territoire, fondée sur une véritable politique de développement régional et une solidarité territoriale établie entre régions riches et régions pauvres. Les régions centrales de Montréal et de Québec qui ont été maintenues à l'écart de cet événement n'ont pu apporter leur contribution à cet effort d'actualisation et de redéfinition du développement régional, ni créer l'amorce d'un dialogue en faveur d'une politique territoriale plus sensible aux besoins des régions non centrales.

Dans certains milieux, on affirme d'emblée que les coûts d'une politique d'occupation dynamique du territoire en régions périphériques et intermédiaires atteindraient des niveaux impossibles à supporter par l'État et moins encore par les populations et organisations économiques des régions centrales. C'est conclure bien rapidement un débat qui n'a pas eu lieu. Sa tenue aurait le mérite de soulever cinq grandes questions et de dégager des élé- ments de réponses, sinon des réactions de nature à nourrir la réflexion devant conduire à la formulation de choix, préalables à l'élaboration de la politique territoriale la plus appropriée: 1) Souhaitons-nous comme société occuper d'une façon durable le territoire actuellement habité des régions périphériques et intermédiaires et pourquoi ? 2) Comment percevons-nous cette occupation du territoire? 3)Peut-on évaluer les coûts de l'occupation dynamique du territoire habité dans un perspective durable? 4) Jusqu'où sommes-nous prêts à consentir les ressources et les efforts requis? et enfin, 5) Quelles stratégies à court, moyen et long termes doiton mettre de l'avant pour assurer l'atteinte de cette grande finalité de société ?

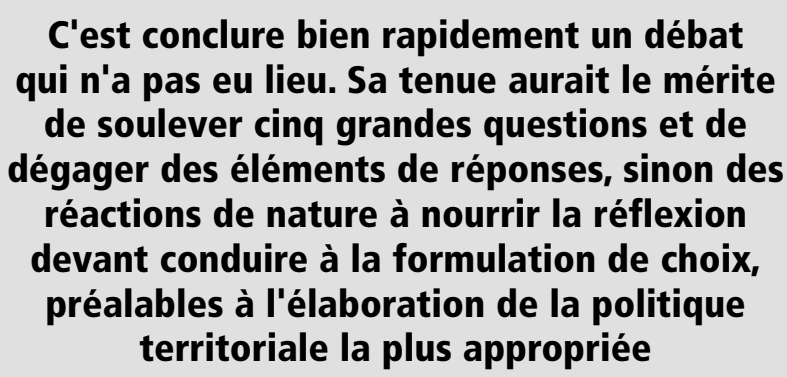

L'ancien maire de Montréal, Pierre Bourque, promoteur du concept «Une île, une ville» et artisan de la relance économique de Montréal, déclarait récemment que «Montréal, qui était en zone dévastée il y a sept ou huit ans, a maintenant pris son envol et il n'est plus nécessaire d'y intervenir comme autrefois. Ce sont les régions éloignées qui ont besoin d'un plus grand soutien de l'État pour réaliser des projets structurants $»^{6}$. Il n'est peut-être pas seul à penser de la sorte.

\section{Conclusion}

Sur le terrain, nombreux sont les directeurs d'organismes de développement, agents de développement et animateurs communautaires qui ressentent l'essoufflement et vivent les frustrations liées aux limites de leur action. Les situations sont de plus en plus criantes, les demandes de plus en plus pressantes et les perspectives réalistes de changer le cours des choses, de moins en moins évidentes. Avec la maturité acquise de quelques années de pratique, ils réalisent l'ampleur et la complexité des problèmes et la portée bien limitée des politiques et stratégies appliquées et des ressources dont ils disposent pour stopper le dépérissement et engager les communautés en péril sur la voie du développement durable. 
Dans un contexte où l'environnement est propice à la croissance, le développement local peut faire des merveilles par ses actions d'information, de sensibilisation, de mobilisation, de formation et d'accompagnement à la concrétisation de projets. En l'absence de ce contexte, aux dimensions tant sociales qu'économiques, les efforts, la ténacité, l'imagination peuvent, certes, faire émerger des projets mais généralement d'une ampleur insuffisante en nombre, en taille et en diversité pour contrer le processus de dévitalisation économique et démographique en cours depuis plus de 40 ans... bien que des miracles soient toujours possibles.

Exercé dans les conditions actuelles dans les régions périphériques et intermédiaires, le développement local est une opération gagnante: gagnante pour les gouvernements centraux car à entretenir l'espoir on contient la frustration, l'agitation, le désordre et la révolte; gagnante aussi pour le modèle de la concentration et la thèse des Higgins, Martin et Raynaud du début des années 1970 qui prônaient la consolidation de quelques pôles centraux, principalement Montréal (la locomotive économique du Québec), au détriment d'une aide substantielle aux régions. Cette thèse inspire toujours la philosophie territoriale des gouvernements qui se succèdent depuis, et que la pratique du développement local vient conforter en oeuvrant auprès des territoires et communautés « résiduels» en détresse.

Exercé dans les conditions actuelles dans les régions périphériques et intermédiaires, le développement local est une opération gagnante: gagnante pour les gouvernements centraux car à entretenir l'espoir on contient la frustration, l'agitation, le désordre et la révolte; gagnante aussi pour le modèle de la concentration et la thèse des Higgins, Martin et Raynaud du début des années 1970 qui prônaient la consolidation de quelques pôles centraux, principalement Montréal (la locomotive économique du Québec), au détriment d'une aide substantielle aux régions. Cette thèse inspire toujours la philosophie territoriale des gouvernements qui se succèdent depuis, et que la pratique du développement local vient conforter en oeuvrant auprès des territoires et communautés " résiduels " en détresse.
Comme on a tenté de l'exposer, la fragilité des régions périphériques et intermédiaires, et plus particulièrement des communautés rurales qui les composent, est un mal structurel dont la cure ne peut être trouvée dans les seules médecines locales. Des interventions majeures d'une extrême dextérité et assurance sont devenues nécessaires. Celles-ci ne sont pas évidentes. L'exercice visant à les préciser se bute à des carences majeures dans la connaissance du tableau d'ensemble et risque d'errer ou de verser dans l'utopie tant que l'état des lieux n'aura pas fait l'objet d'une mise à jour complète; tant que les causes exogènes du déclin régional et local ne feront pas l'objet d'une plus grande attention et d'une volonté réelle de s'y attaquer; tant que des ressources appropriées et d'envergure ne seront pas consenties; tant que de nouveaux consensus n'auront pas été convenus entre les différents partenaires politiques, économiques et civils interpellés par les crises territoriales. Ces consensus méritent d'ailleurs de bénéficier de l'éclairage d'approches et d'idées nouvelles. Voilà un chantier bien stimulant pour la jeune génération de chercheurs régionalistes et ruralistes.

\section{Des interventions majeures d'une extrême dextérité et assurance sont devenues nécessaires.}

Notes et références

1 Bernard Vachon est détenteur d'un doctorat et spécialiste en développement régional et local. Il est également professeur associé au Département de géographie de l'Université du Québec à Montréal.

2 Benko, Georges et Alain Lipietz (sous la direction de) (1992). Les régions qui gagnent. Districts et réseaux: les nouveaux paradigmes de la géographie économique. Paris, Presses universitaires de France, 424 p.

3 SADC: Société d'aide au développement des collectivités. Il s'agit d'une structure d'intervention locale qui relève du gouvernement fédéral. Au nombre de 54 au Québec, elles sont nées de la fusion des Comités d'aide au développement des collectivités et des Centres d'aide aux entreprises, antérieurs d'une dizaine d'années aux CLD.

4 CLD: Comité local de développement. Structure d'intervention locale qui relève du gouvernement provincial. Mis sur pied en 1997, il sont plus d'une centaine actuellement.

5 Solidarité rurale du Québec: Organisme conseil auprès du gouvernement en matière de ruralité. Responsable de la formation et de l'encadrement des agents ruraux (plus de 115). Créé à la suite des États généraux du monde rural en 1991.

6 Le Soleil, 15 février 2003 